\title{
Guest Editorial to the Special Issue on MoDELS 2006
}

\author{
Oscar Nierstrasz • Jon Whittle
}

Published online: 7 August 2008

(C) Springer-Verlag 2008

This issue of "Software and Systems Modeling" and the following one are devoted to selected papers of the ninth MODELS Conference held in Genoa, Italy, from October 16,2006 . The conference has established itself as one of the key international venues for the presentation of scientific results in the domain of model-driven engineering and related topics such as software modeling and model transformation.

A total of 51 papers were selected for publication in the conference proceedings from 178 papers submitted for review from 34 different countries. As usual, both technical and experience papers were accepted, and separate program subcommittees evaluated the two kinds of submissions.

There were two keynote speakers, Hassan Gomaa, who spoke about Architecture-Centric Real-Time Systems and Product Lines, and Irun R. Cohen, who spoke on Immune System Computation and the Immunological Homunculus. Both speakers kindly provided invited papers for the the conference proceedings.

The conference also included three panels on diverse topics, twelve workshops, including an Educators' Symposium and a Doctoral Symposium, and six tutorials on topics ranging from model-driven development to software factories.

After the conclusion of the conference, the program committee was asked to propose a selection of the best papers for publication in a special issue of SoSyM. In the end, eleven papers were proposed, and their authors were invited

\section{O. Nierstrasz ( $\square)$}

IAM-Inst. Informatik u. Angewandte Mathematik, Universität Bern, Neubrückstr 10, 3012 Bern, Switzerland e-mail: oscar@iam.unibe.ch

\section{J. Whittle}

Department of Computing,

Lancaster University, Bailrigg,

Lancaster, Lancs LA1 4YW, UK

e-mail:whittle@comp.lancs.ac.uk to submit extended versions of their conference papers to this special issue. All submitted papers underwent a full review process, and were evaluated by at least three reviewers, generally including both some of the original PC members and some fresh reviewers. Eight papers were finally selected for inclusion in SoSyM, distributed over two SoSyM issues.

The first six papers are included in this issue, and the remaining two will appear in the next issue.

"Correctly Defined Concrete Syntax" by Thomas Baar presents an approach for defining concrete syntax of visual languages and checking this definition for correctness and absence of ambiguities.

"Semantics of OCL Specified with $Q V T$ " by Slavisa Markovic and Thomas Baar proposes an approach based on metamodeling and model transformation to formalize the semantics of the Object Constraint Language of the UML.

"Model-Driven Analysis and Synthesis of Textual Concrete Syntax" by Pierre-Alain Muller, Franck Fleurey, Fréderic Fondement, Michel Hassenforder, Rémi Schneckenburger, Sebastien Gerard and Jean-Marc Jézéquel describes an approach for specifying the concrete syntax of textual languages whose abstract syntax has been specified by a metamodel. The specification can be used for both analysis and synthesis. By exploiting bi-directional mappings, ambiguities are avoided when transforming from models to text and vice versa.

"Understanding and Improving UML Package Merge" by Juergen Dingel, Zinovy Diskin and Alanna Zito presents a detailed analysis of package merge, as it is used in the UML 2 specification. The analysis focuses on identifying ambiguities, inconsistencies, and gaps in the current understanding and specification of package merge, as well as offering solutions to some of the problems that have been identified.

"Improving the definition of general constraints in UML" by Dolors Costal, Cristina Gómez, Anna Queralt, Ruth 
Raventós and Ernest Teniente uses constraint stereotypes to facilitate the specification of frequently used general constraints as predefined constraints.

"Model-Driven Assessment of System Dependability" by Sadaf Mustafiz, Ximeng Sun, Joerg Kienzle and Hans Vangheluwe presents a model-based approach to enable developers to analyze the dependability of use cases by means of a probabilistic extension of statecharts to model interaction requirements.

"Meta-Environment and Executable Meta-Language using Smalltalk" by Stéphane Ducasse, Tudor Girba, Adrian
Kuhn and Lukas Renggli is an experience report showing how Smalltalk can be used as an executable meta-language for software modeling and reengineering (paper to appear in SoSym issue 1, 2009).

Finally, "From Model Transformation to Incremental Bidirectional Model Synchronization" by Robert Wagner and Holger Giese presents a novel approach to incremental model transformation based on triple graph grammars (paper to appear in SoSyM issue 1, 2009). 